\title{
3D-printable cell crowding device enables imaging of live cells in compression
}

\author{
Liam P Dow', Aimal H Khankhel', John Abram² \& Megan T Valentine*,2
}

\begin{abstract}
We designed and fabricated, using low-cost 3D printing technologies, a device that enables direct control of cell density in epithelial monolayers. The device operates by varying the tension of a silicone substrate upon which the cells are adhered. Multiple devices can be manufactured easily and placed in any standard incubator. This allows long-term culturing of cells on pretensioned substrates until the user decreases the tension, thereby inducing compressive forces in plane and subsequent instantaneous cell crowding. Moreover, the low-profile device is completely portable and can be mounted directly onto an inverted optical microscope. This enables visualization of the morphology and dynamics of living cells in stretched or compressed conditions using a wide range of high-resolution microscopy techniques.
\end{abstract}

\section{METHOD SUMMARY}

We used 3D printing technologies to create a mechanical testing device that enables long-term culture of cells on pretensioned substrates that can be returned to zero stress under user command. The devices are compatible with a variety of imaging platforms, enabling real-time visualization of the dynamic response of cells to in-plane compression.

\section{KEYWORDS}

3D printing $\cdot$ cell compression - epithelial mechanics - mechanobiology $\cdot$ microscopy

${ }^{1}$ Biomolecular Science \& Engineering Program, University of California, Santa Barbara, CA 93106, USA; ${ }^{2}$ Department of Mechanical Engineering, University of California, Santa Barbara, CA 93106, USA; *Author for correspondence: valentine@ engineering.ucsb.edu

BioTechniques 68: 275-278 (May 2020) 10.2144/btn-2019-0160
Epithelial homeostasis requires the precise regulation of cell density and has been found to be controlled by mechanical intercellular forces within the monolayer [1]. In particular, when crowded above the target density, cells experience intercellular compressive forces and undergo mechanically influenced live-cell extrusion [1-5]. Failure to extrude can result in tumorigenesis, while hyperextrusion can compromise barrier function. Therefore, studies of epithelial cell mechanics and mechanobiology are critical to understanding healthy tissue maintenance and also how dysfunction of cell migration can lead to cancer and other diseases [6].

Unfortunately, there are limited experimental tools to enable such studies. Several commercial cell-stretching systems are available (e.g., from Flexcell International, NC, USA; Strex, CA, USA; CellScale, Ontario, Canada) and a number of custom-built devices using extensible silicone [7-12], piezoelectric [13], pneumatic [14-17] and recently dielectric actuation [18] have been reported. However, microscopy studies of crowding-induced live-cell extrusion are particularly demanding: cells must be cultured on stretched membranes for hours to days to reach confluency before crowding is induced and then must be imaged at high spatiotemporal resolution to observe their morphology and dynamics. Although some current devices are amenable to imaging $[8,10,11,15,16,19]$, it is not possible to maintain loading conditions continuously in culture or to move the cells in a loaded state between the incubator and the microscope. This severely limits analysis of the dynamic changes that occur before, during and after cell extrusion.

Here, we report a new approach based on the design of low-cost and completely portable cell stretching devices that allow culture of cells on pretensioned substrates and that can be easily transferred to imaging platforms without any changes in cell loading. Moreover, the ease and low cost of manufacturing (typically < $\$ 300$ ) allows for the simultaneous use of multiple devices.

To meet the needs of dynamic extrusion measurements, we applied the following constraints: the device must be: compatible with the existing microscope stage and apertures (here, the Leica TCS SP8 laser scanning confocal fluorescence microscope); capable of imposing uniform, repeatable compressive strains up to $30 \%$; able to be placed within a cell incubator and easily transported to/from the microscope facility; liquid-tight to ensure that culture medium does not leak during transport or imaging; and amenable to sterilization. Two additional constraints are that the device must position the cells within the desired focal depth and imaging must occur directly through a glass coverslip.

To customize the device, we employed in-house fused deposition modeling style 3D printing with a Stratasys $\mathrm{f} 270$ printer. F123 acrylonitrile butadiene styrene build material was chosen due to its high resistance to thermal deformation, ease of printing and cost-effectiveness. Quick support release was used as the support material. Following printing of the necessary device components, the device was assembled per the instructions provided in the Supplemental materials. All components were purchased from McMaster-Carr or 3D printed, except for the double-sided lead screw, for which a portion of the purchased threaded rod was manually reduced and rethreaded.

In operation, the user first secures both ends of a rectangular silicone substrate between the two clamps (Figure 1), while threading the central portion of the substrate over the indenter. The indenter serves as a height reference for the substrate position, ensuring that the cells, which are adhered to the bottom surface of the substrate, are positioned within the objective focal depth. Several fiducial markers spaced $20 \mathrm{~mm}$ apart were engraved into the base of the device to allow the user to quickly apply a predetermined strain to the substrate. To 
(A)

(B)
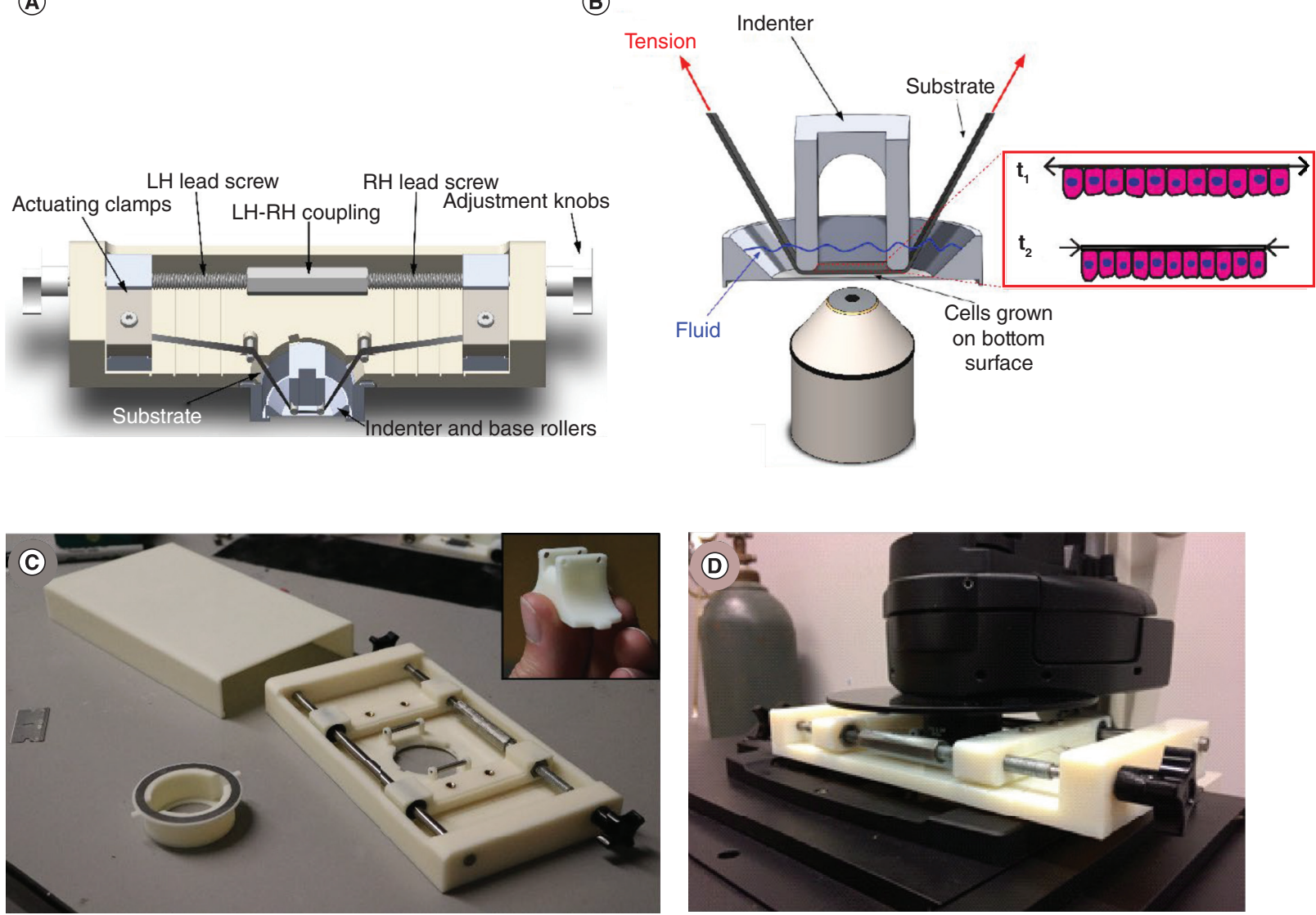

Figure 1. Device design and construction compatible for long-term cell culture and live-cell imaging. (A) Schematic of device in cross-section with the substrate in tension. (B) Lead screw rotation induces substrate compression. (C) Image of assembled device with custom fit lid. (D) Device mounted on a common stage holder of an inverted fluorescence microscope in operation.

LH: Left hand; RH: Right hand.

provide an aqueous environment for the epithelium, a 35-mm glass-bottomed dish (MatTek) containing medium was held magnetically by a 3D-printed holder just below the substrate.

Tension within the substrate can be varied by turning a double-sided lead screw, ensuring that both clamps move outward (or inward) simultaneously and that cells located in the imaging region are not significantly laterally displaced during testing. On the opposite side of the device, a guide rail serves as a support for the actuating clamps. Linear bearings between the clamps and the guide rail provide smooth motion while two sets of stainless-steel rollers supporting the substrate reduce friction to improve uniformity of the applied strain. The lead screw rotation is fully reversible, providing compression or tension to the substrate as desired. To ensure selfcontainment and minimize contamination, a lid can be attached to the top side of the device. Images and schematics of the completed device are provided in Figure 1; see Supplementary materials for additional schematics.

To determine the extent and uniformity of the strain within the membrane, we used digital image correlation to visualize the strain field (Figure 2). A random uniform distribution of speckles was spray-painted onto the silicone rubber; the painted strip was then loaded into the device and imaged under various states of strain. We measured proportional strain levels respective to revolutions of the lead screw, which averaged into a linear relationship across different strain states (Figure 2C). Full experimental details are provided in the Supplementary materials.

To demonstrate the utility of this device for measurements of cell dynamics under compression, we used a model epithelial system of Madin-Darby Canine Kidney II cells expressing eGFP E-cadherin [20]. To promote cell attachment, the silicone rubber substrate was first functionalized with fibronectin (see Supplementary materials for details) before being placed within the cell compression device and pretensioned as desired. Once the functionalized substrate was pretensioned, the device was inverted and approximately 50,000 cells were deposited onto the surface over the indenter. To ensure cell adherence, the inverted device was then covered with parafilm and placed in the incubator for $3 \mathrm{~h}$. After incubation, the device was flipped and the adhered cells were rinsed with phosphate-buffered saline before being submerged in the mediumfilled MatTek dish. The custom 3D-printed lid was then fitted to the device, which was transported to the incubator for culture until confluency was achieved (usually after 1-2 days). 
(A)
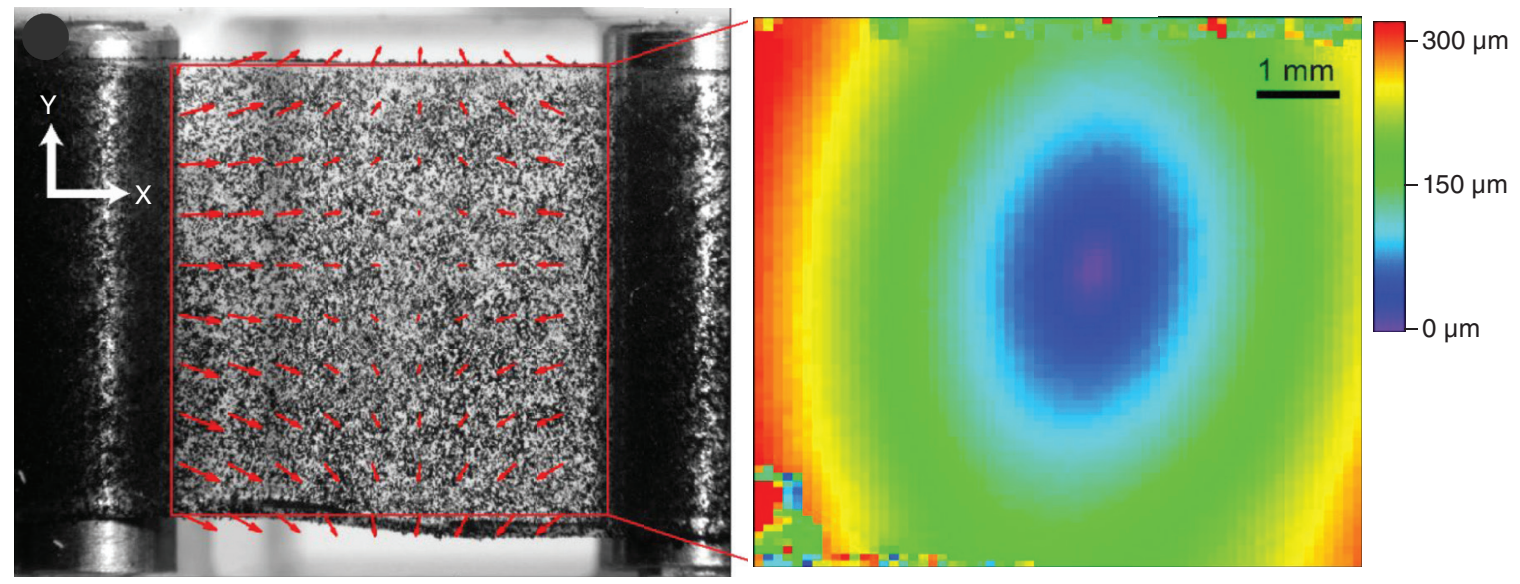

(B)
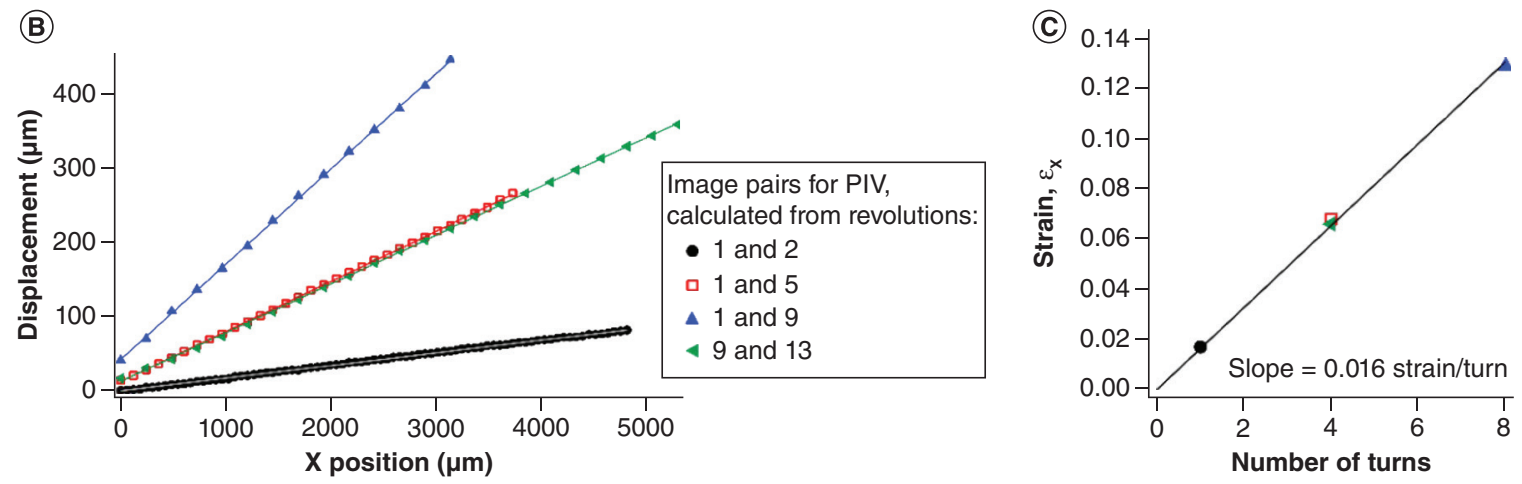

Figure 2. Digital image correlation demonstrates and quantifies linear strain of substrate. (A) Representative mapping of pixel displacement direction (left, arrows in foreground) and magnitude (right) obtained through the analysis of images of the speckled silicone rubber substrate (left, background). (B) The average lateral displacement as a function of $x$-position was quantified through the pairwise comparison of images obtained across different tension states. The numbers in the legend refer to the number of revolutions of the outer knob turning the lead screw (see Supplementary materials for details) for the two images forming the pair; the slope of each line gives the dimensionless substrate strain, $\varepsilon_{x^{\prime}}$ per turn, shown in (C).

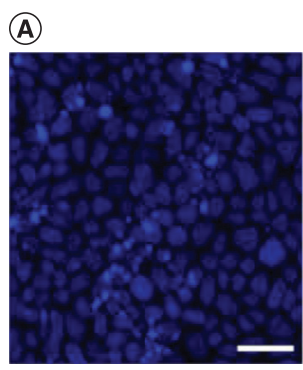

(B)

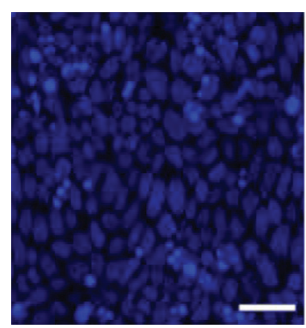

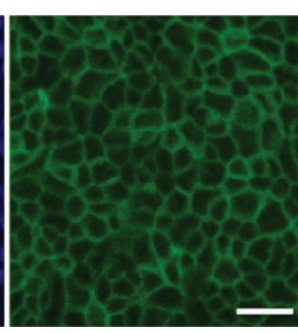

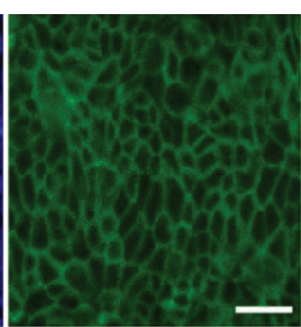

(C)
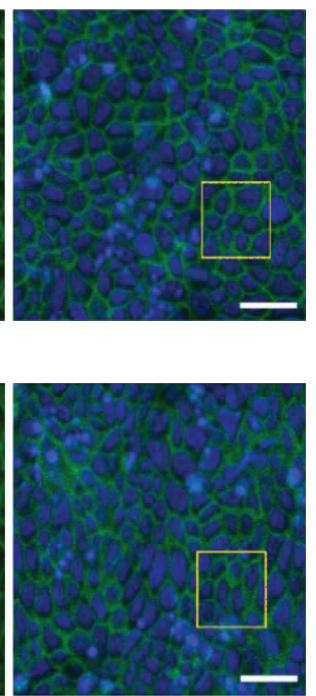
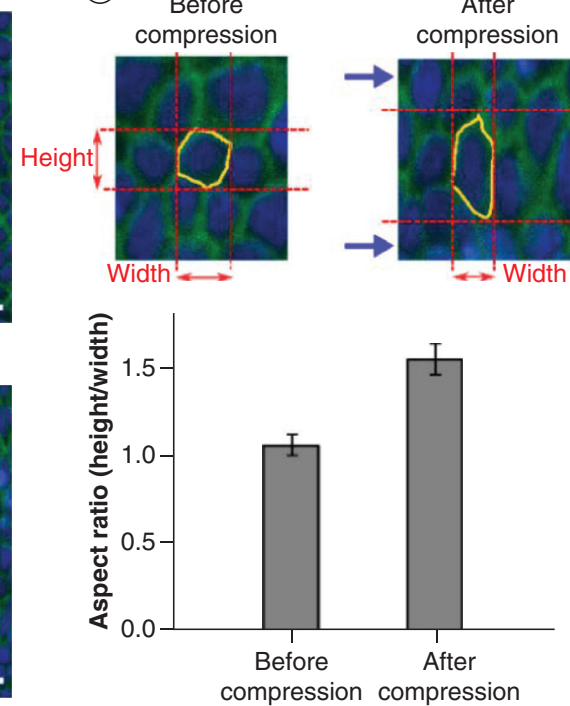

After

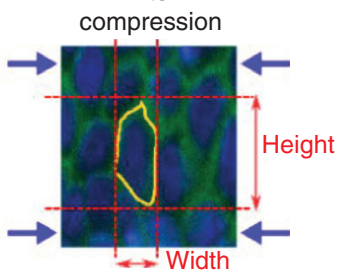

mpression compression

Figure 3. Substrate compression successfully crowds cells for live-cell extrusion studies. (A) Uncompressed and (B) compressed epithelial cell monolayer cultured on a pretensioned (prestrain value of 30\%) silicone rubber substrate within the 3D-printed compression device (nuclei: blue, Hoechst; E-cadherin: green, eGFP; far right are merged channels). Scale bar is $30 \mu \mathrm{m}$. (C) Zoomed-in regions of cells before and after compression. Bar graph shows mean values of aspect ratio as measured from 40 randomly selected cells from each of panels $A$ and $B$. Error bars indicate standard error of the mean. 
Once cells reached confluency, the device was transferred to the microscopy facility, where the tension was released by the desired amount and the compressed cells were imaged using an inverted Leica TCS SP8 spectral confocal microscope with a 10× air objective (Figure 3). Before compression, the cell aspect ratio (height/ width) was $1.07 \pm 0.06$, indicating no preferred orientation within the plane of the substrate. Upon compression ( $30 \%$ strain), the aspect ratio increased to $1.56 \pm 0.09$, due to a reduction in cell size along the compression axis and an elongation in the orthogonal axis. We also measured an approximate $20 \%$ increase in cell density along the compression axis (Supplementary Figure 1).

In summary, we designed, manufactured and tested a low-cost portable cell compression device amenable to both long-term culturing and imaging by microscopy. This innovation enables visualization of the morphology and dynamics of living cells in stretched or compressed conditions using a wide range of high-resolution microscopy techniques.

\section{SUPPLEMENTARY DATA}

To view the supplementary data that accompany this paper please visit the journal website at: www.future-science. com/doi/suppl/10.2144/btn-2019-0160

\section{AUTHOR CONTRIBUTIONS}

AH Khankhel and MT Valentine conceived of the project. J Abram and LP Dow designed, manufactured and tested devices. LP Dow and $\mathrm{AH}$ Khankhel performed biological assays. MT Valentine supervised the project. LP Dow and MT Valentine analyzed data and LP Dow, MT Valentine and J Abram wrote the manuscript.

\section{ACKNOWLEDGMENTS}

We thank S Streichan for the kind gift of the E-Cadherin-GFP transfected MDCK cells, D Bothman for the helpful advice in design, and $Y$ Kwon for providing mechanical data. We acknowledge the use of CNSI Innovation Workshop, Biological Nanostructures Laboratory, and Microfluidics Facility, all of the UCSB California NanoSystems Institute (CNSI) as well as use of the UCSB NRI-MCDB Microscopy Facility.

\section{FINANCIAL \& COMPETING INTERESTS DISCLOSURE}

This work was supported by the US National Science Foundation (NSF) through a Graduate Research Fellowship to $\mathrm{AH}$ Khankhel and partially supported by NSF Award CMMI-1254893 (to MT Valentine) and by the Harold $G$ and Leila $Y$ Mathers Foundation (to MT Valentine). LP Dow was supported by the UCSB Biomolecular Science and Engineering Regents Fellowship. The Leica TCS SP8 Resonant Scanning Confocal Microscope was acquired by MT Valentine under NSF Award DBI-1625770. The authors have no other relevant affiliations or financial involvement with any organization or entity with a financial interest in or financial conflict with the subject matter or materials discussed in the manuscript apart from those disclosed.

No writing assistance was utilized in the production of this manuscript.

\section{OPEN ACCESS}

This work is licensed under the AttributionNonCommercial-NoDerivatives 4.0 Unported License. To view a copy of this license, visit http://creativecommons.org/licenses/ by-nc-nd/4.0/

\section{REFERENCES}

1. Gudipaty SA, Lindblom J, Loftus PD et al. Mechanical stretch triggers rapid epithelial cell division through Piezo1. Nature 543(7643), 118-121 (2017).

2. Eisenhoffer GT, Rosenblatt J. Bringing balance by force: live cell extrusion controls epithelial cell numbers. Trends Cell Biol. 23(4), 185-192 (2013).

3. Saw TB, Doostmohammadi A, Nier V et al. Topological defects in epithelia govern cell death and extrusion. Nature 544(7649), 212-216 (2017)

4. Kocgozlu L, Saw TB, Le AP et al. Epithelial cell packing induces distinct modes of cell extrusions. Curr. Biol. 26(21) 2942-2950 (2016)

5. Eisenhoffer GT, Loftus PD, Yoshigi M et al. Crowding induces live cell extrusion to maintain homeostatic cell numbers in epithelia. Nature 484(7395), 546-549 (2012).

6. Gudipaty SA, Rosenblatt J. Epithelial cell extrusion: pathways and pathologies. Semin. Cell Dev. Biol. 67, 132-140 (2017).

7. Ursekar CP, Teo S-K, Hirata H, Harada I, Chiam K-H Sawada Y. Design and construction of an equibiaxial cell stretching system that is improved for biochemical analysis. PLOS ONE 9(3), e90665 (2014).

8. Shao $Y$, Tan $X$, Novitski $R$ et al. Uniaxial cell stretching device for live-cell imaging of mechanosensitive cellular functions. Rev. Sci. Instrum. 84(11), 114304-1-114304-8 (2013)

9. Tremblay D, Chagnon-Lessard S, Mirzaei M, Pelling AE, Godin M. A microscale anisotropic biaxial cell stretching device for applications in mechanobiology. Biotechnol. Lett. 36(3), 657-665 (2014).

10. Huang L, Mathieu PS, Helmke BP. A stretching device for high-resolution live-cell imaging. Ann. Biomed. Eng. 38(5) 1728-1740 (2010).

11. Dan A, Huang RB, Leckband DE. Dynamic imaging reveals coordinate effects of cyclic stretch and substrate stiffness on endothelial integrity. Ann. Biomed. Eng. 44(12), 3655-3667 (2016)

12. Sigaut $L$, Von Bilderling $C$, Bianchi M, Burdisso JE Gastaldi L, Pietrasanta LI. Live cell imaging reveals focal adhesions manoresponses in mammary epithelial 9788: 1-15 (2018)

13. Clark WW, Smith R, Janes K, Winkler J, Mulcahy M. Development of a piezoelectrically actuated cell stretching device. In: Smart Structures and Materials 2000: Industrial and Commercial Applications of Smart Structures Technologies. SPIE, CA, USA, 294-301 (2000).

14. Kreutzer J, Ikonen L, Hirvonen J, Pekkanen-Mattila M, Aalto-Setälä K, Kallio P. Pneumatic cell stretching system for cardiac differentiation and culture. Med. Eng. Phys. 36(4), 496-501 (2014).

15. Huang $Y$, Nguyen NT. A polymeric cell stretching device for real-time imaging with optical microscopy. Biomed Microdevices 15(6), 1043-1054 (2013)

16. Shimizu K, Shunori A, Morimoto K, Hashida M, Konishi $\mathrm{S}$. Development of a biochip with serially connected . Deveric balloons for cell-stretching culture. Actuators, B Chem. 156(1), 486-493 (2011).

17. Sato $K$, Nitta M, Ogawa A. A microfluidic cell stretch device to investigate the effects of stretching stress on artery smooth muscle cell proliferation in pulmonary arterial hypertension. Inventions 4(1), 1 (2018).

18. Li Z, Gao C, Fan S et al. Cell nanomechanics based on dielectric elastomer actuator device. Nano-Micro Lett. 11(1) 98: 1-19 (2019).

19. Wyatt TPJ, Fouchard J, Lisica A et al. Actomyosin controls planarity and folding of epithelia in response to compression. Nat. Mater. 19(1), 109-117 (2020).

20. Puliafito A, Hufnagel L, Neveu $P$ et al. Collective and single cell behavior in epithelial contact inhibition. Proc. Natl Acad. Sci. USA 109(3), 739-744 (2012). 